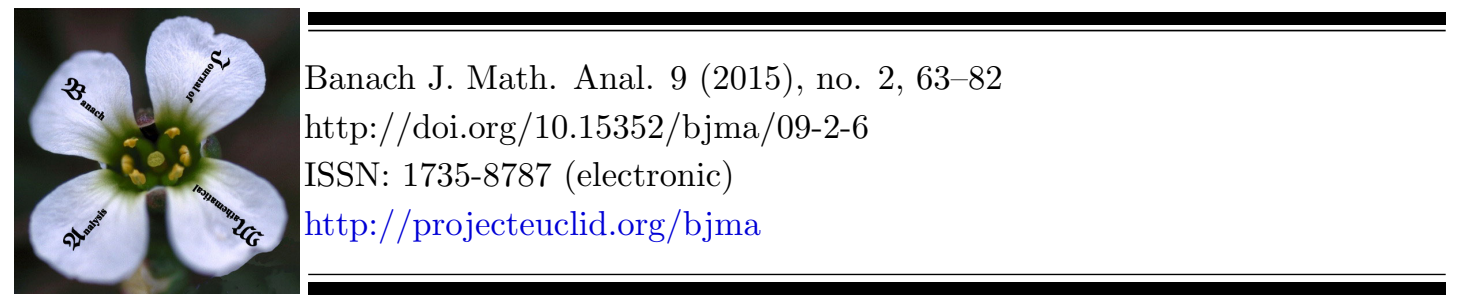

\title{
ON SOME NEW CONVERSES OF CONVEX INEQUALITIES IN HILBERT SPACE
}

\author{
ROZARIJA JAKŠIĆ* AND JOSIP PEČARIĆ \\ Communicated by M. Fujii
}

\begin{abstract}
In this paper we study some new converses of the operator version for the Jensen inequality and the scalar Lah-Ribarič inequality for continuous convex functions and we will give applications of those results to quasiarithmetic means and power means for selfadjoint operators. Furthermore, we will also give improvements of the obtained results.
\end{abstract}

\section{INTRODUCTION AND PRELIMINARIES}

The Jensen inequality for convex functions plays a very important role in the Theory of Inequalities due to the fact that it implies the whole series of the other classical inequalities such as the quasi-arithmetic mean and arithmetic mean inequalities, Hölder and Minkowski inequalities, Ky Fan's inequality etc.

One of the most famous ones amongst them is the so called Lah-Ribarič inequality, which we state in the following theorem.

Theorem 1.1. ([10]) Let $f:[m, M] \rightarrow \mathbb{R}$ be a convex function, $y_{1}, \cdots, y_{n} \in$ $[m, M]$ real numbers and let $p_{1}, \cdots, p_{n}$ be nonnegative real numbers such that $P_{n}=\sum_{i=1}^{n} p_{i}>0$. Then

$$
\frac{1}{P_{n}} \sum_{i=1}^{n} p_{i} f\left(y_{i}\right) \leq \frac{M-\bar{y}}{M-m} f(m)+\frac{\bar{y}-m}{M-m} f(M),
$$

Date: Received: Mar. 26, 2014.; Revised: Jun. 2, 2014.; Accepted: Jun. 17, 2014.

* Corresponding author.

2010 Mathematics Subject Classification. Primary 47A63; Secondary 47A64, 47C99.

Key words and phrases. Selfadjoint operators, Jensen's inequality, Lah-Ribarič's inequality, convex functions, means. 
where $\bar{y}=\frac{1}{P_{n}} \sum_{i=1}^{n} p_{i} y_{i}$.

It was obtained in 1973. by P. Lah and M. Ribarič in their paper [10]. Since then, there have been many papers written on the subject of it's generalizations and converses.

In this paper we refer to a general form of the Jensen inequality for selfadjoint operators. In order to present our results, we first need to introduce the appropriate settings.

Let $H$ be a Hilbert space and let $\mathcal{B}(H)$ be the $C^{*}$-algebra of all bounded (i.e., continuous) linear operators on $H$. A bounded linear operator $A$ on a Hilbert space $H$ is said to be selfadjoint if $A=A^{*}$. An operator $A \in \mathcal{B}(H)$ is selfadjoint if and only if $\langle A x, x\rangle \in \mathbb{R}$ for every $x \in H$. We denote by $\mathcal{B}_{h}(H)$ a semi-space of all selfadjoint operators in $\mathcal{B}(H)$. If $A$ is a selfadjoint operator and $f$ is a real valued continuous function on $S p(A)$, then $f(t) \geq 0$ for every $t \in S p(A)$ implies that $f(A) \geq 0$, i.e., $f(A)$ is a positive operator on $H$. Equivalently, if both $f$ and $g$ are real valued continuous function on $S p(A)$, then the following property holds:

$$
f(t) \geq g(t) \text { for any } t \in S p(A) \text { implies that } f(A) \geq g(A)
$$

in the operator order of $\mathcal{B}(H)$.

The result stated below provides an operator version for Jensen's inequality and it is due to Mond and Pečarić [15] (see also [3]):

Theorem 1.2. ([15]) (Mond - Pečarić) Let $A \in \mathcal{B}_{h}(H)$ be a selfadjoint operator with $S p(A) \subseteq[m, M]$ for some scalars $m<M$. If $f$ is a convex function on $[m, M]$, then

$$
f(\langle A x, x\rangle) \leq\langle f(A) x, x\rangle
$$

for each $x \in H$ with $\|x\|=1$.

Jensen's type inequalities for selfadjoint operators in Hilbert spaces have been an object of study for many different papers. For more on the Mond-Pečarić inequality, i.e. Jensen's inequality for selfadjoint operators and it's refinements, converses and applications, see [1], [4], [5], [6], [11], [12] and [13].

R. Jakšic and J. Pečarić in their paper [7] gave the following result, which concerns the difference between the right and the left side of the Mond-Pečarić inequality (1.3).

Theorem 1.3. ([7]) Let $A \in \mathcal{B}_{h}(H)$ be a selfadjoint operator with $S p(A) \subseteq$ $[m, M]$ for some scalars $m<M$. If $f$ is a continuous convex function on an interval of real numbers $I$ such that $[m, M] \subset \stackrel{\circ}{I}$, where $\stackrel{\circ}{I}$ is the interior of $I$, then

$$
\begin{aligned}
0 & \leq\langle f(A) x, x\rangle-f(\langle A x, x\rangle) \\
& \leq(M-\langle A x, x\rangle)(\langle A x, x\rangle-m) \frac{f_{-}^{\prime}(M)-f_{+}^{\prime}(m)}{M-m} \\
& \leq \frac{1}{4}(M-m)\left(f_{-}^{\prime}(M)-f_{+}^{\prime}(m)\right)
\end{aligned}
$$

holds for each unit vector $x$ in $H$. If $f$ is concave, the inequalities in (1.4) are reversed. 
We also need the following converse for the Mond-Pečarić inequality that provides a generalization of the Lah-Ribarič inequality for convex functions stated earlier:

Theorem 1.4. ([15]) Let $A$ be a selfadjoint operator on the Hilbert space $H$ and assume that $S p(A) \subseteq[m, M]$ for some scalars $m$ and $M$ with $m<M$. If $f$ is a convex function on $[m, M]$, then

$$
\langle f(A) x, x\rangle \leq \frac{M-\langle A x, x\rangle}{M-m} f(m)+\frac{\langle A x, x\rangle-m}{M-m} f(M)
$$

for each $x \in H$ with $\|x\|=1$.

Remark 1.5. One can see that if in Theorem 1.4 we choose $A=\operatorname{diag}\left(\mathrm{y}_{1}, \cdots, \mathrm{y}_{\mathrm{n}}\right)$ and $x=\frac{1}{\sqrt{P_{n}}}\left(\sqrt{p_{1}}, \cdots, \sqrt{p_{n}}\right)$, where $y_{i}, p_{i}(i=1, \cdots, n)$ and $P_{n}$ are defined in Theorem 1.1, then the inequality (1.5) becomes exactly (1.1).

The authors in [7] also gave a series of inequalities that concerns with the difference between the right and the left side of the inequality (1.5), which we state below.

Theorem 1.6. ([7]) Let the assumptions of Theorem 1.3 hold. If $f$ is a continuous convex function on an interval of real numbers $I$ such that $[m, M] \subset \stackrel{\circ}{I}$, where $\stackrel{\circ}{I}$ is the interior of $I$, then

$$
\begin{aligned}
0 & \leq \frac{M-\langle A x, x\rangle}{M-m} f(m)+\frac{\langle A x, x\rangle-m}{M-m} f(M)-\langle f(A) x, x\rangle \\
& \leq \frac{f_{-}^{\prime}(M)-f_{+}^{\prime}(m)}{M-m}\left\langle\left(M 1_{H}-A\right)\left(A-m 1_{H}\right) x, x\right\rangle \\
& \leq \frac{f_{-}^{\prime}(M)-f_{+}^{\prime}(m)}{M-m}(M-\langle A x, x\rangle)(\langle A x, x\rangle-m) \\
& \leq \frac{1}{4}(M-m)\left(f_{-}^{\prime}(M)-f_{+}^{\prime}(m)\right)
\end{aligned}
$$

holds for each unit vector $x$ in $H$. If $f$ is concave, the inequalities in (1.6) are reversed.

The main aim of this paper is to give refinements and improvements of converses of the Jensen inequality (1.4) and the scalar Lah-Ribarič inequality (1.6) for selfadjoint operators obtained in [7].

In the next section, in Theorem 2.1 we shall give a refinement of the inequality (1.4) and in Theorem 2.4 we shall give two similar series of inequalities that refine the inequality (1.6). Also, we shall give applications of the obtained results to quasi-arithmetic means and power means of selfadjoint operators.

In the last section, in Theorem 5.2 we shall improve the results from Theorem 2.1, and show that we have obtained more accurate upper bounds for the difference between left and right side of the scalar Jensen inequality for selfadjoint operators (1.3). We shall also obtain an improvement of the scalar Lah-Ribarič inequality for selfadjoint operators (1.5), and in Theorem 5.7 we shall improve the results from Theorem 2.4, and show that we have obtained more accurate lower 
bound for the difference between left and right side of the scalar Lah-Ribarič inequality for selfadjoint operators (1.5)

\section{MAin RESUlts}

Our first result is a refinement of the converse of Jensen's inequality (1.4) for selfadjoint operators obtained in [7].

Theorem 2.1. Let $A \in \mathcal{B}_{h}(H)$ be a selfadjoint operator with $\operatorname{Sp}(A) \subseteq[m, M]$ for some scalars $m<M$. If $f$ is a continuous convex function on an interval of real numbers $I$ such that $[m, M] \subset \stackrel{\circ}{I}$, where $\stackrel{\circ}{I}$ is the interior of $I$ and $x \in H$ such that $\|x\|=1$, then

$$
\begin{aligned}
0 & \leq\langle f(A) x, x\rangle-f(\langle A x, x\rangle) \\
& \leq(M-\langle A x, x\rangle)(\langle A x, x\rangle-m) \sup _{t \in\langle m, M\rangle} \Psi_{f}(t ; m, M) \\
& \leq(M-\langle A x, x\rangle)(\langle A x, x\rangle-m) \frac{f_{-}^{\prime}(M)-f_{+}^{\prime}(m)}{M-m} \\
& \leq \frac{1}{4}(M-m)\left(f_{-}^{\prime}(M)-f_{+}^{\prime}(m)\right) .
\end{aligned}
$$

We also have the inequalities

$$
\begin{aligned}
0 & \leq\langle f(A) x, x\rangle-f(\langle A x, x\rangle) \leq \frac{1}{4}(M-m)^{2} \Psi_{f}(\langle A x, x\rangle ; m, M) \\
& \leq \frac{1}{4}(M-m)\left(f_{-}^{\prime}(M)-f_{+}^{\prime}(m)\right),
\end{aligned}
$$

where $\Psi_{f}(\cdot ; m, M):\langle m, M\rangle \rightarrow \mathbb{R}$ is defined by

$$
\Psi_{f}(t ; m, M)=\frac{1}{M-m}\left(\frac{f(M)-f(t)}{M-t}-\frac{f(t)-f(m)}{t-m}\right) .
$$

If $f$ is concave, the inequalities are reversed.

Proof. First we assume that $f$ is convex. If $\langle A x, x\rangle=m$ or $\langle A x, x\rangle=M$, the inequalities are clear. Let us suppose that $\langle A x, x\rangle \in\langle m, M\rangle$.

The first inequality follows directly from (1.3). By Theorem 1.4, we have

$$
\begin{aligned}
\langle f(A) & x, x\rangle-f(\langle A x, x\rangle) \leq \frac{M-\langle A x, x\rangle}{M-m} f(m)+\frac{\langle A x, x\rangle-m}{M-m} f(M)-f(\langle A x, x\rangle) \\
& =\frac{(M-\langle A x, x\rangle)(\langle A x, x\rangle-m)}{M-m}\left\{\frac{f(M)-f(\langle A x, x\rangle)}{M-\langle A x, x\rangle}-\frac{f(\langle A x, x\rangle)-f(m)}{\langle A x, x\rangle-m}\right\} \\
& =(M-\langle A x, x\rangle)(\langle A x, x\rangle-m) \Psi_{f}(\langle A x, x\rangle ; m, M) \\
& \leq(M-\langle A x, x\rangle)(\langle A x, x\rangle-m) \sup _{t \in\langle m, M\rangle} \Psi_{f}(t ; m, M),
\end{aligned}
$$


so we have proved the second inequality in (2.1).

$$
\begin{aligned}
\sup _{t \in\langle m, M\rangle} \Psi_{f}(t ; m, M) & =\frac{1}{M-m} \sup _{t \in\langle m, M\rangle}\left\{\frac{f(M)-f(t)}{M-t}-\frac{f(t)-f(m)}{t-m}\right\} \\
& \leq \frac{1}{M-m}\left(\sup _{t \in\langle m, M\rangle} \frac{f(M)-f(t)}{M-t}+\sup _{t \in\langle m, M\rangle} \frac{-(f(t)-f(m))}{t-m}\right) \\
& =\frac{1}{M-m}\left(\sup _{t \in\langle m, M\rangle} \frac{f(M)-f(t)}{M-t}-\inf _{t \in\langle m, M\rangle} \frac{f(t)-f(m)}{t-m}\right) \\
& =\frac{f_{-}^{\prime}(M)-f_{+}^{\prime}(m)}{M-m},
\end{aligned}
$$

which proves the third inequality in (2.1). To prove the last inequality in (2.1), we notice that for every $t \in[m, M]$, the inequality $\frac{(M-t)(t-m)}{M-m} \leq \frac{1}{4}(M-m)$ is valid. Since $\langle A x, x\rangle \in[m, M]$, we can replace $t \leftrightarrow\langle A x, x\rangle$ and the proof is completed.

The proof of the inequalities (2.2) is clear from the proof of the inequalities (2.1). If $f$ is concave, then $-f$ is convex, so we can apply (2.1) and (2.2) to function $-f$ and obtain the reversed inequalities for $f$.

Remark 2.2. Observe that the function $\Psi_{f}(\cdot ; m, M):\langle m, M\rangle \rightarrow \mathbb{R}$ defined in (2.3) is actually the second order divided difference of the function $f$ at the points $\mathrm{m}$, $\mathrm{t}$ and $\mathrm{M}$ for any $t \in\langle m, M\rangle$.

In order to obtain a converse of the Lah-Ribarič inequality for convex functions (1.6), we need the following result found in [7]:

Lemma 2.3. ([7]) Let $f$ be a continuous convex function on an interval of real numbers $I$ and $m, M \in \mathbb{R}, m<M$ with $[m, M] \subset \stackrel{\circ}{\perp}$, where $\stackrel{\circ}{I}$ is the interior of $I$. Then for any $t \in[m, M]$ the following inequalities are valid:

$$
\begin{aligned}
\Delta_{f}(t ; m, M) & =\frac{(t-m) f(M)+(M-t) f(m)}{M-m}-f(t) \\
& \leq(M-t)(t-m) \sup _{t \in\langle m, M\rangle} \Psi_{f}(t ; m, M) \\
& \leq \frac{(M-t)(t-m)}{M-m}\left(f_{-}^{\prime}(M)-f_{+}^{\prime}(m)\right) \\
& \leq \frac{1}{4}(M-m)\left(f_{-}^{\prime}(M)-f_{+}^{\prime}(m)\right) .
\end{aligned}
$$

Also we have

$$
\Delta_{f}(t ; m, M) \leq \frac{1}{4}(M-m)^{2} \Psi_{f}(t ; m, M) \leq \frac{1}{4}(M-m)\left(f_{-}^{\prime}(M)-f_{+}^{\prime}(m)\right),
$$

where $\Psi_{f}(\cdot ; m, M):\langle m, M\rangle \rightarrow \mathbb{R}$ is defined in (2.3). If $f$ is concave, the inequalities are reversed.

Theorem 2.4. Let the assumptions of Theorem 2.1 hold. If $f$ is a continuous convex function on an interval of real numbers $I$ such that $[m, M] \subset \stackrel{\circ}{I}$, where $\stackrel{\circ}{I}$ is 
the interior of $I$ and $x \in H$ such that $\|x\|=1$, then the following inequalities are valid

$$
\begin{aligned}
0 & \leq \frac{(\langle A x, x\rangle-m) f(M)+(M-\langle A x, x\rangle) f(m)}{M-m}-\langle f(A) x, x\rangle \\
& \leq\left\langle\left(M 1_{H}-A\right)\left(A-m 1_{H}\right) x, x\right\rangle \sup _{t \in\langle m, M\rangle} \Psi_{f}(t ; m, M) \\
& \leq \frac{\left\langle\left(M 1_{H}-A\right)\left(A-m 1_{H}\right) x, x\right\rangle}{M-m}\left(f_{-}^{\prime}(M)-f_{+}^{\prime}(m)\right) \\
& \leq \frac{(M-\langle A x, x\rangle)(\langle A x, x\rangle-m)}{M-m}\left(f_{-}^{\prime}(M)-f_{+}^{\prime}(m)\right) \\
& \leq \frac{1}{4}(M-m)\left(f_{-}^{\prime}(M)-f_{+}^{\prime}(m)\right)
\end{aligned}
$$

(ii)

$$
\begin{aligned}
0 & \leq \frac{(\langle A x, x\rangle-m) f(M)+(M-\langle A x, x\rangle) f(m)}{M-m}-\langle f(A) x, x\rangle \\
& \leq\left\langle\left(M 1_{H}-A\right)\left(A-m 1_{H}\right) x, x\right\rangle \sup _{t \in\langle m, M\rangle} \Psi_{f}(t ; m, M) \\
& \leq(M-\langle A x, x\rangle)(\langle A x, x\rangle-m) \sup _{t \in\langle m, M\rangle} \Psi_{f}(t ; m, M) \\
& \leq \frac{(M-\langle A x, x\rangle)(\langle A x, x\rangle-m)}{M-m}\left(f_{-}^{\prime}(M)-f_{+}^{\prime}(m)\right) \\
& \leq \frac{1}{4}(M-m)\left(f_{-}^{\prime}(M)-f_{+}^{\prime}(m)\right)
\end{aligned}
$$

where $\Psi_{f}(\cdot ; m, M):\langle m, M\rangle \rightarrow \mathbb{R}$ is defined in (2.3). If $f$ is concave, the inequalities are reversed.

Proof. Let us assume that $f$ is convex. If $\langle A x, x\rangle=m$ or $\langle A x, x\rangle=M$, the inequalities are clear. Let us suppose that $\langle A x, x\rangle \in\langle m, M\rangle$.

The first inequalities follow directly from Theorem 1.4. Due to property (1.2), we can replace $t$ with operator $A$ in the inequalities (2.4) from Lemma 2.3 and obtain

$$
\begin{aligned}
& \frac{1}{M-m}\left(\left(A-m 1_{H}\right) f(M)+\left(M 1_{H}-A\right) f(m)\right)-f(A) \\
& \leq\left(M 1_{H}-A\right)\left(A-m 1_{H}\right) \sup _{t \in\langle m, M\rangle} \Psi_{f}(t ; m, M) \\
& \leq \frac{1}{M-m}\left(M 1_{H}-A\right)\left(A-m 1_{H}\right)\left(f_{-}^{\prime}(M)-f_{+}^{\prime}(m)\right) \\
& \leq \frac{1}{4}(M-m)\left(f_{-}^{\prime}(M)-f_{+}^{\prime}(m)\right) 1_{H}
\end{aligned}
$$

in the operator order of $\mathcal{B}(H)$.

Because scalar product is linear in the first argument and $\|x\|=1$, by applying 
it to $(2.7)$ we have

$$
\begin{aligned}
& \frac{\langle A x, x\rangle-m}{M}-m(M)+\frac{M-\langle A x, x\rangle}{M-m} f(m)-\langle f(A) x, x\rangle \\
& \quad \leq\left\langle\left(M 1_{H}-A\right)\left(A-m 1_{H}\right) x, x\right\rangle \sup _{t \in\langle m, M\rangle} \Psi_{f}(t ; m, M) \\
& \quad \leq \frac{1}{M-m}\left\langle\left(M 1_{H}-A\right)\left(A-m 1_{H}\right) x, x\right\rangle\left(f_{-}^{\prime}(M)-f_{+}^{\prime}(m)\right) .
\end{aligned}
$$

Since $h(t)=(M-t)(t-m)$ is concave, from the Jensen inequality we have

$$
\left\langle\left(M 1_{H}-A\right)\left(A-m 1_{H}\right) x, x\right\rangle \leq(M-\langle A x, x\rangle)(\langle A x, x\rangle-m) .
$$

To prove the last inequality in (2.5), we notice that for every $t \in[m, M]$, $\frac{(M-t)(t-m)}{M-m} \leq \frac{1}{4}(M-m)$ is valid, and since $\langle A x, x\rangle \in[m, M]$ for every $x \in H$ such that $\|x\|=1$, we can replace $t$ with $\langle A x, x\rangle$.

The first and second inequalities in (2.6) are just the first and second inequalities in (2.5). Again, the function $g(t)=(M-t)(t-m)$ is concave, so from Jessen's inequality it follows that $\left\langle\left(M 1_{H}-A\right)\left(A-m 1_{H}\right) x, x\right\rangle \leq(M-\langle A x, x\rangle)(\langle A x, x\rangle-m)$, which proves the third inequality in (2.6). In the proof of Theorem 2.1 we have shown that the inequality $\sup _{t \in\langle m, M\rangle} \Psi_{f}(t ; m, M) \leq \frac{f_{-}^{\prime}(M)-f_{+}^{\prime}(m)}{M-m}$ is valid, so the fourth inequality in (2.6) directly follows. To prove the last inequality in (2.6), we notice that for every $t \in[m, M]$, the inequality $\frac{(M-t)(t-m)}{M-m} \leq \frac{1}{4}(M-m)$ is valid. Since $\langle A x, x\rangle \in[m, M]$, we have $\frac{(M-\langle A x, x\rangle)(\langle A x, x\rangle-m)}{M-m} \leq \frac{1}{4}(M-m)$ and thus the proof is complete.

If $f$ is concave, then $-f$ is convex, so we can apply (2.5) and (2.6) to function $-f$ and obtain reversed inequalities for $f$.

Remark 2.5. For an extensive list of results in inequalities for functions of selfadjoint operators related to Mond-Pečarić method and applications, see [2] and the references therein.

\section{Applications to QUASI-ARITHMETIC MEANS}

Let $A$ be a positive invertible operator on a Hilbert space such that $S p(A) \subseteq$ $[m, M]$ for some scalars $m<M$ and $x$ a unit vector in $H$. Let $f$ be a strictly monotone continuous function on $[m, M]$. Quasi-arithmetic mean of the operator $A$ with respect to $f$ is defined by

$$
M_{f}(A, x)=f^{-1}(\langle f(A) x, x\rangle) .
$$

In this section, the objective is to apply the results from the previous section to some strictly monotone functions, and in that way get two series of inequalities involving quasi-arithmetic means.

Theorem 3.1. Let $A \in \mathcal{B}_{h}(H)$ be a selfadjoint operator with $\operatorname{Sp}(A) \subseteq[m, M]$ for some scalars $m<M$, let $f, g$ be strictly monotone continuous functions on an interval of real numbers I such that $[m, M] \subset \stackrel{\circ}{I}$, where $\stackrel{\circ}{I}$ is the interior of $I$, 
and let us denote $\left[m_{g}, M_{g}\right]=g([m, M])$. If the function $f \circ g^{-1}$ is convex, then for each $x \in H$ such that $\|x\|=1$ we have

$$
\begin{aligned}
0 & \leq f\left(M_{f}(A, x)\right)-f\left(M_{g}(A, x)\right) \\
& \leq\left(M_{g}-\langle g(A) x, x\rangle\right)\left(\langle g(A) x, x\rangle-m_{g}\right) \sup _{t \in\langle m, M\rangle} \Psi_{f \circ g^{-1}}\left(g(t) ; m_{g}, M_{g}\right) \\
& \leq\left(M_{g}-\langle g(A) x, x\rangle\right)\left(\langle g(A) x, x\rangle-m_{g}\right) \frac{\left(f \circ g^{-1}\right)_{-}^{\prime}\left(M_{g}\right)-\left(f \circ g^{-1}\right)_{+}^{\prime}\left(m_{g}\right)}{M_{g}-m_{g}} \\
& \leq \frac{1}{4}\left(M_{g}-m_{g}\right)\left(\left(f \circ g^{-1}\right)_{-}^{\prime}\left(M_{g}\right)-\left(f \circ g^{-1}\right)_{+}^{\prime}\left(m_{g}\right)\right) .
\end{aligned}
$$

We also have the inequalities

$$
\begin{aligned}
0 & \leq f\left(M_{f}(A, x)\right)-f\left(M_{g}(A, x)\right) \leq \frac{1}{4}\left(M_{g}-m_{g}\right)^{2} \Psi_{f \circ g^{-1}}\left(\langle g(A) x, x\rangle ; m_{g}, M_{g}\right) \\
& \leq \frac{1}{4}\left(M_{g}-m_{g}\right)\left(\left(f \circ g^{-1}\right)_{-}^{\prime}\left(M_{g}\right)-\left(f \circ g^{-1}\right)_{+}^{\prime}\left(m_{g}\right)\right) .
\end{aligned}
$$

If $f \circ g^{-1}$ is concave, then the inequalities are reversed.

Proof. Function $f \circ g^{-1}$ is obviously continuous. Let us assume that $f \circ g^{-1}$ is convex. If $g$ is strictly increasing, then $m_{g}=g(m), M_{g}=g(M)$; if $g$ is strictly decreasing, then $m_{g}=g(M), M_{g}=g(m)$. The conditions of Theorem 2.1 are satisfied, so we can obtain (3.2) and (3.3) by substituting $f \leftrightarrow f \circ g^{-1}$ and $A \leftrightarrow g(A)$ in $(2.1)$ and $(2.2)$.

Now let us assume that $f \circ g^{-1}$ is concave. Then the function $-f \circ g^{-1}$ is convex, so we can obtain reversed inequalities by replacing $f \circ g^{-1}$ with $-f \circ g^{-1}$.

Theorem 3.2. Under the assumptions from Theorem 3.1, if the function $f \circ g^{-1}$ is convex, then the following inequalities are valid:

$$
\begin{aligned}
0 & \leq \frac{M_{g}-\langle g(A) x, x\rangle}{M_{g}-m_{g}} f(m)+\frac{\langle g(A) x, x\rangle-m_{g}}{M_{g}-m_{g}} f(M)-f\left(M_{f}(A, x)\right) \\
& \leq\left\langle\left(M_{g} 1_{H}-g(A)\right)\left(g(A)-m_{g} 1_{H}\right) x, x\right\rangle \sup _{t \in\langle m, M\rangle} \Psi_{f \circ g^{-1}}\left(g(t) ; m_{g}, M_{g}\right) \\
& \leq \frac{\left\langle\left(M_{g} 1_{H}-g(A)\right)\left(g(A)-m_{g} 1_{H}\right) x, x\right\rangle}{M_{g}-m_{g}}\left(\left(f \circ g^{-1}\right)_{-}^{\prime}\left(M_{g}\right)-\left(f \circ g^{-1}\right)_{+}^{\prime}\left(m_{g}\right)\right) \\
& \leq \frac{\left(M_{g}-\langle g(A) x, x\rangle\right)\left(\langle g(A) x, x\rangle-m_{g}\right)}{M_{g}-m_{g}}\left(\left(f \circ g^{-1}\right)_{-}^{\prime}\left(M_{g}\right)-\left(f \circ g^{-1}\right)_{+}^{\prime}\left(m_{g}\right)\right) \\
& \leq \frac{1}{4}\left(M_{g}-m_{g}\right)\left(\left(f \circ g^{-1}\right)_{-}^{\prime}\left(M_{g}\right)-\left(f \circ g^{-1}\right)_{+}^{\prime}\left(m_{g}\right)\right)
\end{aligned}
$$


(ii)

$$
\begin{aligned}
0 & \leq \frac{M_{g}-\langle g(A) x, x\rangle}{M_{g}-m_{g}} f(m)+\frac{\langle g(A) x, x\rangle-m_{g}}{M_{g}-m_{g}} f(M)-f\left(M_{f}(A, x)\right) \\
& \leq\left\langle\left(M_{g} 1_{H}-g(A)\right)\left(g(A)-m_{g} 1_{H}\right) x, x\right\rangle \sup _{t \in\langle m, M\rangle} \Psi_{f \circ g^{-1}}\left(g(t) ; m_{g}, M_{g}\right) \\
& \leq\left(M_{g}-\langle g(A) x, x\rangle\right)\left(\langle g(A) x, x\rangle-m_{g}\right) \sup _{t \in\langle m, M\rangle} \Psi_{f \circ g^{-1}}\left(g(t) ; m_{g}, M_{g}\right) \\
& \leq \frac{\left(M_{g}-\langle g(A) x, x\rangle\right)\left(\langle g(A) x, x\rangle-m_{g}\right)}{M_{g}-m_{g}}\left(\left(f \circ g^{-1}\right)_{-}^{\prime}\left(M_{g}\right)-\left(f \circ g^{-1}\right)_{+}^{\prime}\left(m_{g}\right)\right) \\
& \leq \frac{1}{4}\left(M_{g}-m_{g}\right)\left(\left(f \circ g^{-1}\right)_{-}^{\prime}\left(M_{g}\right)-\left(f \circ g^{-1}\right)_{+}^{\prime}\left(m_{g}\right)\right)
\end{aligned}
$$

for each $x \in H$ such that $\|x\|=1$, where $\left[m_{g}, M_{g}\right]=g([m, M])$. If $f \circ g^{-1}$ is concave, the inequalities are reversed.

Proof. Similarly to the proof of the previous theorem, let us assume that $f \circ g^{-1}$ is convex (obviously, $f \circ g^{-1}$ is continuous).

If $g$ is strictly increasing, then $m_{g}=g(m), M_{g}=g(M)$; if $g$ is strictly decreasing, then $m_{g}=g(M), M_{g}=g(m)$. The conditions of Theorem 2.4 are satisfied. We obtain (3.4) and (3.5) by substituting $f \leftrightarrow f \circ g^{-1}$ and $A \leftrightarrow g(A)$ in (2.5) and (2.6).

Now let us assume that $f \circ g^{-1}$ is concave. Then the function $-f \circ g^{-1}$ is convex, so we can obtain reversed inequalities by replacing $f \circ g^{-1}$ with $-f \circ g^{-1}$.

Remark 3.3. Let $f, g, x$ and $A$ be as in Theorem 3.2. We can rewrite the first inequality in (3.4) as:

$$
(g(M)-g(m)) f\left(M_{f}(A, x)\right)-(f(M)-f(m)) g\left(M_{g}(A, x)\right) \leq g(M) f(m)-g(m) f(M)
$$

if $f \circ g^{-1}$ is convex and $g$ is strictly increasing. If $f \circ g^{-1}$ is concave, the inequality is reversed.

\section{Applications to power means}

Let $A$ be a positive invertible operator on a Hilbert space and $x$ a unit vector in $H$. For $r \in \mathbb{R}$, the power mean $M_{r}(A, x)$ is defined by

$$
M_{r}(A, x)=\left(\left\langle A^{r} x, x\right\rangle\right)^{1 / r} .
$$

In [15, p.69] it has been shown that if $r \rightarrow 0$, then $\left(\left\langle A^{r} x, x\right\rangle\right)^{1 / r}$ converges monotone to $\exp \langle\log A x, x\rangle$, so we can extend the definition of the power mean to the case $r=0$.

Power means are a special case of quasi-arithmetic means defined in (3.1), so series of inequalities involving arithmetics means analogue to those from the previous section easily follow from Theorem 3.1 and Theorem 3.2 respectively.

Theorem 4.1. Let $A \in \mathcal{B}_{h}(H)$ be a selfadjoint operator with $S p(A) \subseteq[m, M]$ for some scalars $0<m<M<\infty$. Let $x$ be a unit vector in $H, r, s \in \mathbb{R}$ such 
that $r<s$ and let

$$
\phi_{s, t}(t)= \begin{cases}t^{s / r} & : r \neq 0, s \neq 0 \\ \frac{1}{r} \log t & : r \neq 0, s=0 \\ e^{s t} & : r=0, s \neq 0\end{cases}
$$

If $r<0<s$ or $0<r<s$, then

$$
\begin{aligned}
0 & \leq\left(M_{s}(A, x)\right)^{s}-\left(M_{r}(A, x)\right)^{s} \\
& \leq\left(M^{r}-\left\langle A^{r} x, x\right\rangle\right)\left(\left\langle A^{r} x, x\right\rangle-m^{r}\right) \sup _{t \in\langle m, M\rangle} \Psi_{\phi_{s, t}}\left(t^{r} ; m^{r}, M^{r}\right) \\
& \leq \frac{s}{r}\left(M^{r}-\left\langle A^{r} x, x\right\rangle\right)\left(\left\langle A^{r} x, x\right\rangle-m^{r}\right) \frac{M^{s-r}-m^{s-r}}{M^{r}-m^{r}} \\
& \leq \frac{s}{4 r}\left(M^{r}-m^{r}\right)\left(M^{s-r}-m^{s-r}\right),
\end{aligned}
$$

and we also have

$$
\begin{aligned}
0 & \leq\left(M_{s}(A, x)\right)^{s}-\left(M_{r}(A, x)\right)^{s} \leq \frac{1}{4}\left(M^{r}-m^{r}\right)^{2} \Psi_{\phi_{s, t}}\left(\left\langle A^{r} x, x\right\rangle ; m^{r}, M^{r}\right) \\
& \leq \frac{s}{4 r}\left(M^{r}-m^{r}\right)\left(M^{s-r}-m^{s-r}\right) .
\end{aligned}
$$

If $r<s<0$, then

$$
\begin{aligned}
0 & \geq\left(M_{s}(A, x)\right)^{s}-\left(M_{r}(A, x)\right)^{s} \\
& \geq\left(M^{r}-\left\langle A^{r} x, x\right\rangle\right)\left(\left\langle A^{r} x, x\right\rangle-m^{r}\right) \sup _{t \in\langle m, M\rangle} \Psi_{\phi_{s, t}}\left(t^{r} ; m^{r}, M^{r}\right) \\
& \geq \frac{s}{r}\left(M^{r}-\left\langle A^{r} x, x\right\rangle\right)\left(\left\langle A^{r} x, x\right\rangle-m^{r}\right) \frac{M^{s-r}-m^{s-r}}{M^{r}-m^{r}} \\
& \geq \frac{s}{4 r}\left(M^{r}-m^{r}\right)\left(M^{s-r}-m^{s-r}\right),
\end{aligned}
$$

and we also have

$$
\begin{aligned}
0 & \geq\left(M_{s}(A, x)\right)^{s}-\left(M_{r}(A, x)\right)^{s} \geq \frac{1}{4}\left(M^{r}-m^{r}\right)^{2} \Psi_{\phi_{s, t}}\left(\left\langle A^{r} x, x\right\rangle ; m^{r}, M^{r}\right) \\
& \geq \frac{s}{4 r}\left(M^{r}-m^{r}\right)\left(M^{s-r}-m^{s-r}\right) .
\end{aligned}
$$

If $r=0$ and $s>0$, then

$$
\begin{aligned}
0 & \leq\left(M_{s}(A, x)\right)^{s}-\left(M_{0}(A, x)\right)^{s} \\
& \leq(\log M-\langle\log A x, x\rangle)(\langle\log A x, x\rangle-\log m) \sup _{t \in\langle m, M\rangle} \Psi_{\phi_{s, t}}(\log t ; \log m, \log M) \\
& \leq s(\log M-\langle\log A x, x\rangle)(\langle\log A x, x\rangle-\log m) \frac{M^{s}-m^{s}}{\log M-\log m} \\
& \leq \frac{s}{4}\left(M^{s}-m^{s}\right) \log \frac{M}{m},
\end{aligned}
$$


and we also have

$$
\begin{aligned}
0 & \leq\left(M_{s}(A, x)\right)^{s}-\left(M_{0}(A, x)\right)^{s} \leq \frac{1}{4}\left(\log \frac{M}{m}\right)^{2} \Psi_{\phi_{s, t}}(\langle\log A x, x\rangle ; \log m, \log M) \\
& \leq \frac{s}{4}\left(M^{s}-m^{s}\right) \log \frac{M}{m}
\end{aligned}
$$

If $r<0$ or $s=0$, then

$$
\begin{aligned}
0 & \leq \log \left(M_{0}(A, x)\right)-\log \left(M_{r}(A, x)\right) \\
& \leq\left(M^{r}-\left\langle A^{r} x, x\right\rangle\right)\left(\left\langle A^{r} x, x\right\rangle-m^{r}\right) \sup _{t \in\langle m, M\rangle} \Psi_{\phi_{s, t}}\left(t^{r} ; m^{r}, M^{r}\right) \\
& \leq-\frac{1}{r} \frac{\left(M^{r}-\left\langle A^{r} x, x\right\rangle\right)\left(\left\langle A^{r} x, x\right\rangle-m^{r}\right)}{M^{r} m^{r}} \\
& \leq \frac{1}{4 r}\left(M^{r}-m^{r}\right)\left(M^{-r}-m^{-r}\right),
\end{aligned}
$$

and we also have

$$
\begin{aligned}
0 & \leq \log \left(M_{0}(A, x)\right)-\log \left(M_{r}(A, x)\right) \leq \frac{1}{4}\left(M^{r}-m^{r}\right)^{2} \Psi_{\phi_{s, t}}\left(\left\langle A^{r} x, x\right\rangle ; m^{r}, M^{r}\right) \\
& \leq \frac{1}{4 r}\left(M^{r}-m^{r}\right)\left(M^{-r}-m^{-r}\right)
\end{aligned}
$$

Proof. This theorem follows directly from Theorem 3.1 in four steps.

Put $f(t)=t^{s}$ and $g(t)=t^{r}$ in Theorem 3.1. Then $f \circ g^{-1}$ is convex and $\phi_{s, t}=$ $f \circ g^{-1}$ whenever $0<r<s$ or $r<0<s$. Therefore (4.1) and (4.2) follow easily from (3.2) and (3.3), respectively.

Put $f(t)=-t^{s}$ and $g(t)=t^{r}$ in Theorem 3.1. Then $f \circ g^{-1}$ is convex and $\phi_{s, t}=-f \circ g^{-1}$ whenever $r<s<0$. Therefore (4.3) and (4.4) follow easily from (3.2) and (3.3), respectively.

Put $f(t)=t^{s}$ and $g(t)=\log t$ in Theorem 3.1. Then $f \circ g^{-1}$ is convex and $\phi_{s, t}=f \circ g^{-1}$ whenever $r=0$ and $s>0$. Therefore (4.5) and (4.6) follow easily from (3.2) and (3.3), respectively.

Put $f(t)=\log t$ and $g(t)=t^{r}$ in Theorem 3.1. Then $f \circ g^{-1}$ is convex and $\phi_{s, t}=f \circ g^{-1}$ whenever $r<0$ and $s=0$. Therefore (4.7) and (4.8) follow easily from (3.2) and (3.3), respectively.

Theorem 4.2. Under the same hypothesis as in the previous theorem, if $0<r<s$ or $r<0<s$, then: 
(i)

$$
\begin{aligned}
0 & \leq \frac{M^{r}-\left\langle A^{r} x, x\right\rangle}{M^{r}-m^{r}} m^{s}+\frac{\left\langle A^{r} x, x\right\rangle-m^{r}}{M^{r}-m^{r}} M^{s}-\left(M_{s}(A, x)\right)^{s} \\
& \leq\left\langle\left(M^{r} 1_{H}-A^{r}\right)\left(A^{r}-m^{r} 1_{H}\right) x, x\right\rangle \sup _{t \in\langle m, M\rangle} \Psi_{\phi_{s, t}}\left(t^{r} ; m^{r}, M^{r}\right) \\
& \leq \frac{s}{r} \frac{\left\langle\left(M^{r} 1_{H}-A^{r}\right)\left(A^{r}-m^{r} 1_{H}\right) x, x\right\rangle}{M^{r}-m^{r}}\left(M^{s-r}-m^{s-r}\right) \\
& \leq \frac{s}{r} \frac{\left(M^{r}-\left\langle A^{r} x, x\right\rangle\right)\left(\left\langle A^{r} x, x\right\rangle-m^{r}\right)}{M^{r}-m^{r}}\left(M^{s-r}-m^{s-r}\right) \\
& \leq \frac{s}{4 r}\left(M^{r}-m^{r}\right)\left(M^{s-r}-m^{s-r}\right)
\end{aligned}
$$

(ii)

$$
\begin{aligned}
0 & \leq \frac{M^{r}-\left\langle A^{r} x, x\right\rangle}{M^{r}-m^{r}} m^{s}+\frac{\left\langle A^{r} x, x\right\rangle-m^{r}}{M^{r}-m^{r}} M^{s}-\left(M_{s}(A, x)\right)^{s} \\
& \leq\left\langle\left(M^{r} 1_{H}-A^{r}\right)\left(A^{r}-m^{r} 1_{H}\right) x, x\right\rangle \sup _{t \in\langle m, M\rangle} \Psi_{\phi_{s, t}}\left(t^{r} ; m^{r}, M^{r}\right) \\
& \leq\left(M^{r}-\left\langle A^{r} x, x\right\rangle\right)\left(\left\langle A^{r} x, x\right\rangle-m^{r}\right) \sup _{t \in\langle m, M\rangle} \Psi_{\phi_{s, t}}\left(t^{r} ; m^{r}, M^{r}\right) \\
& \leq \frac{s}{r} \frac{\left(M^{r}-\left\langle A^{r} x, x\right\rangle\right)\left(\left\langle A^{r} x, x\right\rangle-m^{r}\right)}{M^{r}-m^{r}}\left(M^{s-r}-m^{s-r}\right) \\
& \leq \frac{s}{4 r}\left(M^{r}-m^{r}\right)\left(M^{s-r}-m^{s-r}\right) .
\end{aligned}
$$

If $r<s<0$ then:

(i)

$$
\begin{aligned}
0 & \geq \frac{M^{r}-\left\langle A^{r} x, x\right\rangle}{M^{r}-m^{r}} m^{s}+\frac{\left\langle A^{r} x, x\right\rangle-m^{r}}{M^{r}-m^{r}} M^{s}-\left(M_{s}(A, x)\right)^{s} \\
& \geq\left\langle\left(M^{r} 1_{H}-A^{r}\right)\left(A^{r}-m^{r} 1_{H}\right) x, x\right\rangle \sup _{t \in\langle m, M\rangle} \Psi_{\phi_{s, t}}\left(t^{r} ; m^{r}, M^{r}\right) \\
& \geq \frac{s}{r} \frac{\left\langle\left(M^{r} 1_{H}-A^{r}\right)\left(A^{r}-m^{r} 1_{H}\right) x, x\right\rangle}{M^{r}-m^{r}}\left(M^{s-r}-m^{s-r}\right) \\
& \geq \frac{s}{r} \frac{\left(M^{r}-\left\langle A^{r} x, x\right\rangle\right)\left(\left\langle A^{r} x, x\right\rangle-m^{r}\right)}{M^{r}-m^{r}}\left(M^{s-r}-m^{s-r}\right) \\
& \geq \frac{s}{4 r}\left(M^{r}-m^{r}\right)\left(M^{s-r}-m^{s-r}\right)
\end{aligned}
$$


(ii)

$$
\begin{aligned}
0 & \geq \frac{M^{r}-\left\langle A^{r} x, x\right\rangle}{M^{r}-m^{r}} m^{s}+\frac{\left\langle A^{r} x, x\right\rangle-m^{r}}{M^{r}-m^{r}} M^{s}-\left(M_{s}(A, x)\right)^{s} \\
& \geq\left\langle\left(M^{r} 1_{H}-A^{r}\right)\left(A^{r}-m^{r} 1_{H}\right) x, x\right\rangle \sup _{t \in\langle m, M\rangle} \Psi_{\phi_{s, t}}\left(t^{r} ; m^{r}, M^{r}\right) \\
& \geq\left(M^{r}-\left\langle A^{r} x, x\right\rangle\right)\left(\left\langle A^{r} x, x\right\rangle-m^{r}\right) \sup _{t \in\langle m, M\rangle} \Psi_{\phi_{s, t}}\left(t^{r} ; m^{r}, M^{r}\right) \\
& \geq \frac{s}{r} \frac{\left(M^{r}-\left\langle A^{r} x, x\right\rangle\right)\left(\left\langle A^{r} x, x\right\rangle-m^{r}\right)}{M^{r}-m^{r}}\left(M^{s-r}-m^{s-r}\right) \\
& \geq \frac{s}{4 r}\left(M^{r}-m^{r}\right)\left(M^{s-r}-m^{s-r}\right) .
\end{aligned}
$$

If $s=0$ and $r<0$, then:

(i)

$$
\begin{aligned}
0 & \leq \frac{M^{r}-\left\langle A^{r} x, x\right\rangle}{M^{r}-m^{r}} \log m+\frac{\left\langle A^{r} x, x\right\rangle-m^{r}}{M^{r}-m^{r}} \log M-\log \left(M_{0}(A, x)\right) \\
& \leq\left\langle\left(M^{r} 1_{H}-A^{r}\right)\left(A^{r}-m^{r} 1_{H}\right) x, x\right\rangle \sup _{t \in\langle m, M\rangle} \Psi_{\phi_{s, t}}\left(t^{r} ; m^{r}, M^{r}\right) \\
& \leq-\frac{1}{r} \frac{\left\langle\left(M^{r} 1_{H}-A^{r}\right)\left(A^{r}-m^{r} 1_{H}\right) x, x\right\rangle}{M^{r} m^{r}} \\
& \leq-\frac{1}{r} \frac{\left(M^{r}-\left\langle A^{r} x, x\right\rangle\right)\left(\left\langle A^{r} x, x\right\rangle-m^{r}\right)}{M^{r} m^{r}} \\
& \leq \frac{1}{4 r}\left(M^{r}-m^{r}\right)\left(M^{-r}-m^{-r}\right)
\end{aligned}
$$

(ii)

$$
\begin{aligned}
0 & \leq \frac{M^{r}-\left\langle A^{r} x, x\right\rangle}{M^{r}-m^{r}} \log m+\frac{\left\langle A^{r} x, x\right\rangle-m^{r}}{M^{r}-m^{r}} \log M-\log \left(M_{0}(A, x)\right) \\
& \leq\left\langle\left(M^{r} 1_{H}-A^{r}\right)\left(A^{r}-m^{r} 1_{H}\right) x, x\right\rangle \sup _{t \in\langle m, M\rangle} \Psi_{\phi_{s, t}}\left(t^{r} ; m^{r}, M^{r}\right) \\
& \leq\left(M^{r}-\left\langle A^{r} x, x\right\rangle\right)\left(\left\langle A^{r} x, x\right\rangle-m^{r}\right) \sup _{t \in\langle m, M\rangle} \Psi_{\phi_{s, t}}\left(t^{r} ; m^{r}, M^{r}\right) \\
& \leq-\frac{1}{r} \frac{\left(M^{r}-\left\langle A^{r} x, x\right\rangle\right)\left(\left\langle A^{r} x, x\right\rangle-m^{r}\right)}{M^{r} m^{r}} \\
& \leq \frac{1}{4 r}\left(M^{r}-m^{r}\right)\left(M^{-r}-m^{-r}\right) .
\end{aligned}
$$

If $r=0$ and $s>0$, then: 
(i)

$$
\begin{aligned}
0 & \leq \frac{\log M-\langle\log A x, x\rangle}{\log M-\log m} m^{s}+\frac{\langle\log A x, x\rangle-\log m}{\log M-\log m} M^{s}-\left(M_{s}(A, x)\right)^{s} \\
& \leq\left\langle\left(\log M 1_{H}-\log A\right)\left(\log A-\log m 1_{H}\right) x, x\right\rangle \sup _{t \in\langle m, M\rangle} \Psi_{\phi_{s, t}}(\log t ; \log m, \log M) \\
& \leq s \frac{\left\langle\left(\log M 1_{H}-\log A\right)\left(\log A-\log m 1_{H}\right) x, x\right\rangle}{\log M-\log m}\left(M^{s}-m^{s}\right) \\
& \leq s \frac{(\log M-\langle\log A x, x\rangle)(\langle\log A x, x\rangle-\log m)}{\log M-\log m}\left(M^{s}-m^{s}\right) \\
& \leq \frac{s}{4}\left(M^{s}-m^{s}\right) \log \frac{M}{m}
\end{aligned}
$$

$$
\begin{aligned}
0 & \leq \frac{\log M-\langle\log A x, x\rangle}{\log M-\log m} m^{s}+\frac{\langle\log A x, x\rangle-\log m}{\log M-\log m} M^{s}-\left(M_{s}(A, x)\right)^{s} \\
& \leq\left\langle\left(\log M 1_{H}-\log A\right)\left(\log A-\log m 1_{H}\right) x, x\right\rangle \sup _{t \in\langle m, M\rangle} \Psi_{\phi_{s, t}}(\log t ; \log m, \log M) \\
& \leq(\log M-\langle\log A x, x\rangle)(\langle\log A x, x\rangle-\log m) \sup _{t \in\langle m, M\rangle} \Psi_{\phi_{s, t}}(\log t ; \log m, \log M) \\
& \leq s \frac{(\log M-\langle\log A x, x\rangle)(\langle\log A x, x\rangle-\log m)}{\log M-\log m}\left(M^{s}-m^{s}\right) \\
& \leq \frac{s}{4}\left(M^{s}-m^{s}\right) \log \frac{M}{m} .
\end{aligned}
$$

Proof. All the inequalities can be obtained directly from (3.4) and (3.5) by applying the same methods as in the proof of the previous theorem.

Remark 4.3. The following inequalities are obtained directly from Theorem 4.2:

$$
\left(M^{r}-m^{r}\right)\left(M_{s}(A, x)\right)^{s}-\left(M^{s}-m^{s}\right)\left(M_{r}(A, x)\right)^{r} \leq M^{r} m^{s}-m^{r} M^{s}
$$

if $0<r<s$ or $r<0<s$. In case $r<s<0$, the inequality is reversed. Similarly, for $r=0$ we have:

$$
\left(M_{s}(A, x)\right)^{s} \log \frac{M}{m}-\left(M^{s}-m^{s}\right) \log \left(M_{0}(A, x)\right) \leq m^{s} \log M-M^{s} \log m
$$

Remark 4.4. It is easy to see that $M_{r}(A, x)=\left(M_{-r}(A, x)\right)^{-1}$ holds for every unit vector $x \in H$, selfadjoint operator $\mathrm{A}$ and $r \in \mathbb{R}$. Using that result, we can obtain analogue sequences of inequalities from Theorem 4.1 and Theorem 4.2 by replacing $A \leftrightarrow A^{-1},-r \leftrightarrow s$ and $-s \leftrightarrow r$.

Remark 4.5. For more results concerning quasi-arithmetic mean and power mean, and order relations amongst them and arithmetic mean, see for instance [16] and [17].

\section{IMPROVEMENTS}

In this section we will give improvements of Theorem 2.1 and Theorem 2.4. In order to do so, we first need to state a result found in [9]. 
Lemma 5.1. ([9]) Let $f$ be a convex function on $D_{f}, x, y \in D_{f}$ and $p, q \in[0,1]$ such that $p+q=1$. Then

$$
\begin{aligned}
\min \{p, q\}\left[f(x)+f(y)-2 f\left(\frac{x+y}{2}\right)\right] & \leq p f(x)+q f(y)-f(p x+q y) \\
& \leq \max \{p, q\}\left[f(x)+f(y)-2 f\left(\frac{x+y}{2}\right)\right]
\end{aligned}
$$

The following result is an improvement of Theorem 2.1.

Theorem 5.2. Let $A \in \mathcal{B}_{h}(H)$ be a selfadjoint operator with $\operatorname{Sp}(A) \subseteq[m, M]$ for some scalars $m<M$. If $f$ is a continuous convex function on an interval of real numbers $I$ such that $[m, M] \subset \stackrel{\circ}{\perp}$, where $\stackrel{\circ}{I}$ is the interior of $I$ and $x \in H$ such that $\|x\|=1$, then

$$
\begin{aligned}
0 \leq & \langle f(A) x, x\rangle-f(\langle A x, x\rangle) \\
\leq & (M-\langle A x, x\rangle)(\langle A x, x\rangle-m) \sup _{t \in\langle m, M\rangle} \Psi_{f}(t ; m, M) \\
& -\left(\frac{1}{2}-\frac{1}{M-m}\langle\tilde{A} x, x\rangle\right) \delta_{f} \\
\leq & (M-\langle A x, x\rangle)(\langle A x, x\rangle-m) \frac{f_{-}^{\prime}(M)-f_{+}^{\prime}(m)}{M-m}-\left(\frac{1}{2}-\frac{1}{M-m}\langle\tilde{A} x, x\rangle\right) \delta_{f} \\
\leq & \frac{1}{4}(M-m)\left(f_{-}^{\prime}(M)-f_{+}^{\prime}(m)\right)-\left(\frac{1}{2}-\frac{1}{M-m}\langle\tilde{A} x, x\rangle\right) \delta_{f} .
\end{aligned}
$$

We also have the inequalities

$$
\begin{aligned}
0 & \leq\langle f(A) x, x\rangle-f(\langle A x, x\rangle) \\
& \leq \frac{1}{4}(M-m)^{2} \Psi_{f}(\langle A x, x\rangle ; m, M)-\left(\frac{1}{2}-\frac{1}{M-m}\langle\tilde{A} x, x\rangle\right) \delta_{f} \\
& \leq \frac{1}{4}(M-m)\left(f_{-}^{\prime}(M)-f_{+}^{\prime}(m)\right)-\left(\frac{1}{2}-\frac{1}{M-m}\langle\tilde{A} x, x\rangle\right) \delta_{f}
\end{aligned}
$$

where $\Psi_{f}(\cdot ; m, M):\langle m, M\rangle \rightarrow \mathbb{R}$ is defined in (2.3) and

$$
\delta_{f}=f(m)+f(M)-2 f\left(\frac{m+M}{2}\right), \tilde{A}=\left|A-\frac{m+M}{2} 1_{H}\right| .
$$

If $f$ is concave, the inequalities are reversed. 
Proof. First we assume that $f$ is convex. Let $t \in[m, M]$. Since $\frac{t-m}{M-m}, \frac{M-t}{M-m} \in$ $[0,1]$ and $\frac{t-m}{M-m}+\frac{M-t}{M-m}=1$, from (5.1) it follows that

$$
\begin{aligned}
f(t)= & f\left(\frac{M-t}{M-m} m+\frac{t-m}{M-m} M\right) \\
\leq & \frac{M-t}{M-m} f(m)+\frac{t-m}{M-m} f(M) \\
& -\min \left\{\frac{t-m}{M-m}, \frac{M-t}{M-m}\right\}\left[f(m)+f(M)-2 f\left(\frac{m+M}{2}\right)\right] \\
= & \frac{M f(m)-m f(M)}{M-m}+\frac{f(M)-f(m)}{M-m} t-\min \left\{\frac{t-m}{M-m}, \frac{M-t}{M-m}\right\} \delta_{f} \\
= & \frac{M f(m)-m f(M)}{M-m}+\frac{f(M)-f(m)}{M-m} t-\left(\frac{1}{2}-\frac{1}{M-m}\left|t-\frac{m+M}{2}\right|\right) \delta_{f}
\end{aligned}
$$

Due to property (1.2) we can replace $t$ with the operator $A$ in (5.5) and obtain:

$$
\begin{aligned}
f(A) \leq & \frac{M f(m)-m f(M)}{M-m} 1_{H}+\frac{f(M)-f(m)}{M-m} A \\
& -\left(\frac{1}{2} 1_{H}-\frac{1}{M-m}\left|A-\frac{m+M}{2} 1_{H}\right|\right) \delta_{f}
\end{aligned}
$$

Since scalar product is linear in the first argument, if we apply it on (5.6) for $x \in H$ such that $\|x\|=1$ we get

$$
\begin{aligned}
\langle f(A) x, x\rangle \leq & \frac{M-\langle A x, x\rangle}{M-m} f(m)+\frac{\langle A x, x\rangle-f(m)}{M-m} f(M) \\
& -\left(\frac{1}{2}-\frac{1}{M-m}\left\langle\left|A-\frac{m+M}{2} 1_{H}\right| x, x\right\rangle\right) \delta_{f} .
\end{aligned}
$$

The first inequality in (5.2) follows directly from the operator version of Jensen's inequality (1.3).

Now if we subtract $f(\langle A x, x\rangle)$ from both sides of the inequality (5.7) we have

$$
\begin{aligned}
\langle f(A) x, x\rangle-f(\langle A x, x\rangle) & \\
\leq & \frac{M-\langle A x, x\rangle}{M-m} f(m)+\frac{\langle A x, x\rangle-m}{M-m} f(M) \\
& -f(\langle A x, x\rangle)-\left(\frac{1}{2}-\frac{1}{M-m}\langle\tilde{A} x, x\rangle\right) \delta_{f} \\
= & \frac{(M-\langle A x, x\rangle)(\langle A x, x\rangle-m)}{M-m}\left\{\frac{f(M)-f(\langle A x, x\rangle)}{M-\langle A x, x\rangle}-\frac{f(\langle A x, x\rangle)-f(m)}{\langle A x, x\rangle-m}\right\} \\
& -\left(\frac{1}{2}-\frac{1}{M-m}\langle\tilde{A} x, x\rangle\right) \delta_{f} \\
= & (M-\langle A x, x\rangle)(\langle A x, x\rangle-m) \Psi_{f}(\langle A x, x\rangle ; m, M)-\left(\frac{1}{2}-\frac{1}{M-m}\langle\tilde{A} x, x\rangle\right) \delta_{f} \\
\leq & (M-\langle A x, x\rangle)(\langle A x, x\rangle-m) \sup _{t \in\langle m, M\rangle} \Psi_{f}(t ; m, M)-\left(\frac{1}{2}-\frac{1}{M-m}\langle\tilde{A} x, x\rangle\right) \delta_{f},
\end{aligned}
$$


so we have proved the second inequality in (5.2).

In the proof of Theorem 2.1 we have shown that

$$
\sup _{t \in\langle m, M\rangle} \Psi_{f}(t ; m, M) \leq \frac{f_{-}^{\prime}(M)-f_{+}^{\prime}(m)}{M-m},
$$

which in combination with (5.8) proves the third inequality in (5.2). To prove the last inequality in (5.2), we notice that for every $t \in[m, M]$, the inequality $\frac{(M-t)(t-m)}{M-m} \leq \frac{1}{4}(M-m)$ is valid. Since $\langle A x, x\rangle \in[m, M]$, we can replace $t \leftrightarrow\langle A x, x\rangle$ and the proof is completed.

The proof of the inequalities (5.3) is clear from the proof of the inequalities (5.2). If $f$ is concave, then $-f$ is convex, so we can apply (5.2) and (5.3) to function $-f$ and obtain the reversed inequalities.

Remark 5.3. The inequality (5.6) obtained in the proof of the previous theorem is a known result (see for instance [14]), and the proof is given for the sake of completeness.

Remark 5.4. In Theorem 5.2 with $|A|$ is denoted the absolute value of a Hilbert space operator $A$, that is, $|A|=\left(A^{*} A\right)^{\frac{1}{2}}$, where $A^{*}$ stands for the adjoint operator of $A$. Since $A$ is a selfadjoint operator we have $A^{*}=A$, so

$$
\begin{aligned}
\tilde{A} & =\left|A-\frac{m+M}{2} 1_{H}\right|=\left(\left(A-\frac{m+M}{2} 1_{H}\right)^{*}\left(A-\frac{m+M}{2} 1_{H}\right)\right)^{\frac{1}{2}} \\
& =\left(\left(A-\frac{m+M}{2} 1_{H}\right)\left(A-\frac{m+M}{2} 1_{H}\right)\right)^{\frac{1}{2}} \\
& =\left(\left(A-\frac{m+M}{2} 1_{H}\right)^{2}\right)^{\frac{1}{2}} .
\end{aligned}
$$

Remark 5.5. From $S p(A) \subseteq[m, M]$, that is $m 1_{H} \leq A \leq M 1_{H}$, we easily obtain

$$
-\frac{M-m}{2} 1_{H} \leq A-\frac{m+M}{2} 1_{H} \leq \frac{M-m}{2},
$$

which is

$$
\left(\left(A-\frac{m+M}{2} 1_{H}\right)^{2}\right)^{\frac{1}{2}} \leq \frac{M-m}{2}
$$

so we have $\tilde{A} \leq \frac{M-m}{2}$, and therefore $\langle\tilde{A} x, x\rangle \leq \frac{M-m}{2}$ for any $x \in H$ such that $\|x\|=1$. Now,

$$
\frac{1}{2}-\frac{1}{M-m}\langle\tilde{A} x, x\rangle \geq \frac{1}{2}-\frac{1}{M-m} \frac{M-m}{2}=0 .
$$

Since for a convex function $f$ we have

$$
\delta_{f}=f(m)+f(M)-2 f\left(\frac{m+M}{2}\right) \geq 0,
$$

the following inequality is valid

$$
\left(\frac{1}{2}-\frac{1}{M-m}\langle\tilde{A} x, x\rangle\right) \delta_{f} \geq 0 .
$$


Remark 5.6. Due to inequality (5.9), one can see that under the assumptions from the previous theorem, the inequality (5.7) which we state again

$$
\begin{aligned}
\langle f(A) x, x\rangle \leq & \frac{M-\langle A x, x\rangle}{M-m} f(m)+\frac{\langle A x, x\rangle-f(m)}{M-m} f(M) \\
& -\left(\frac{1}{2}-\frac{1}{M-m}\left\langle\left|A-\frac{m+M}{2} 1_{H}\right| x, x\right\rangle\right) \delta_{f}
\end{aligned}
$$

is an improvement of the scalar Lah-Ribarič inequality for selfadjoint operators (1.5). We can see as well that Theorem 5.2 is really an improvement of Theorem 2.1 obtained in Section 2.

Our next result gives us an improvement of the lower bound from Theorem 2.4 and it is proved in a similar way as the previous theorem by using inequalities (5.7), (5.9) and the operator version of Jensen's inequality (1.3).

Theorem 5.7. Let the assumptions of Theorem 5.2 hold. If $f$ is a continuous convex function on an interval of real numbers $I$ such that $[m, M] \subset \stackrel{\circ}{I}$, where $\stackrel{\circ}{I}$ is the interior of $I$ and $x \in H$ such that $\|x\|=1$, then the following inequalities are valid

$$
\begin{aligned}
0 & \leq\left(\frac{1}{2}-\frac{1}{M-m}\langle\tilde{A} x, x\rangle\right) \delta_{f} \\
& \leq \frac{\langle A x, x\rangle-m}{M-m} f(M)+\frac{M-\langle A x, x\rangle}{M-m} f(m)-\langle f(A) x, x\rangle \\
& \leq\left\langle\left(M 1_{H}-A\right)\left(A-m 1_{H}\right) x, x\right\rangle \sup _{t \in\langle m, M\rangle} \Psi_{f}(t ; m, M) \\
& \leq\left\langle\left(M 1_{H}-A\right)\left(A-m 1_{H}\right) x, x\right\rangle \frac{f_{-}^{\prime}(M)-f_{+}^{\prime}(m)}{M-m} \\
& \leq(M-\langle A x, x\rangle)(\langle A x, x\rangle-m) \frac{f_{-}^{\prime}(M)-f_{+}^{\prime}(m)}{M-m} \\
& \leq \frac{1}{4}(M-m)\left(f_{-}^{\prime}(M)-f_{+}^{\prime}(m)\right)
\end{aligned}
$$

(ii)

$$
\begin{aligned}
0 & \leq\left(\frac{1}{2}-\frac{1}{M-m}\langle\tilde{A} x, x\rangle\right) \delta_{f} \\
& \leq \frac{\langle A x, x\rangle-m}{M-m} f(M)+\frac{M-\langle A x, x\rangle}{M-m} f(m)-\langle f(A) x, x\rangle \\
& \leq\left\langle\left(M 1_{H}-A\right)\left(A-m 1_{H}\right) x, x\right\rangle \sup _{t \in\langle m, M\rangle} \Psi_{f}(t ; m, M) \\
& \leq(M-\langle A x, x\rangle)(\langle A x, x\rangle-m) \sup _{t \in\langle m, M\rangle} \Psi_{f}(t ; m, M) \\
& \leq(M-\langle A x, x\rangle)(\langle A x, x\rangle-m) \frac{f_{-}^{\prime}(M)-f_{+}^{\prime}(m)}{M-m} \\
& \leq \frac{1}{4}(M-m)\left(f_{-}^{\prime}(M)-f_{+}^{\prime}(m)\right)
\end{aligned}
$$


where $\Psi_{f}(\cdot ; m, M):\langle m, M\rangle \rightarrow \mathbb{R}$ is defined in (2.3) and $\tilde{A}$ and $\delta_{f}$ are defined in (5.4). If $f$ is concave, the inequalities are reversed.

Remark 5.8. One can see that by subtracting $\left(\frac{1}{2}-\frac{1}{M-m}\langle\tilde{A} x, x\rangle\right) \delta_{f}$ from all of the inequalities in (5.10) and (5.11) we can obtain an estimate for the difference between left and right side in inequality (5.7).

Theorem 5.2 and Theorem 5.7 can be applied to quasi-arithmetic means and power means in an analogous way as we did with Theorem 2.1 and Theorem 2.4. In that way we can get improvements of the results obtained in Sections 3 and 4.

Acknowledgement. This work has been fully supported by Croatian Science Foundation under the project 5435 .

\section{REFERENCES}

1. P.P. Agarwal and S.S. Dragomir, A survey of Jensen type inequalities for functions of selfadjoint operators in Hilbert space, Comput. Math. Appl. 59, (2010), No 12, 3785-3812.

2. S.S. Dragomir, Hermite-Hadamard's type inequalities for convex functions of selfadjoint operators in Hilbert spaces, Linear Algebra Appl. 436, (2012), 1503-1515.

3. S.S. Dragomir, Some reverses of the Jensen inequality for the functions of selfadjoint operators in Hilbert spaces, J. Inequal. Appl. 2010, Art. ID 496821.

4. S.S. Dragomir, Some Jensen's type inequality for twice differentiable functions of selfadjoint operators in Hilbert spaces, Filomat 23:3, (2009), 211-222.

5. T. Hayashi $A$ note on the Jensen inequality for self-adjoint operators, J. Math. Soc. Japan 62, (2010), No. 3, 949-961.

6. T. Hayashi $A$ note on the Jensen inequality for self-adjoint operators II, J. Math. Soc. Japan 66, (2014), No. 1, 111-121.

7. R. Jakšić and J. Pečarić Converses of convex inequalities in Hilbert space, Rend. Circ. Mat. Palermo 63, (2014), No. 1, 1-9.

8. R. Jakšić and J. Pečarić New converses of Jensen and Lah-Ribarič inequality II, J. Math. Inequal. 7, (2013), No. 4, 617-645.

9. M. Klaričić Bakula, J. Pečarić and J. Perić On the converse Jensen inequality, Appl. Math. Comput. 218, (2012), No.11, 6566-6575.

10. P. Lah and M. Ribarič, Converse of Jensen's inequality for convex functions, Univ. Beograd. Publ. Elektrotehn. Fak. Ser. Mat. Fiz. No. 412-460, 201-205

11. S.M. Malamud, A converse to the Jensen inequality, its matrix extensions and inequalities for minors and eigenvalues, Linear Algebra Appl. 322, (2001), No. 1-3, 19-41.

12. S.M. Malamud, Operator inequalities reverse to the Jensen inequality, Math. Notes 69, (2001), No.4, 582-586.

13. J. Mičić, Y. Seo and S.-E. Takahasi, Inequalities of Furuta and Mond-Pečarić, Math. Inequal. Appl. 2, (1999), 83-111.

14. M.S. Moslehian, J. Mičić and M. Kian, Operator inequalities of Jensen type, Topology Algebra Appl. 1 (2013), 9-21.

15. J. Pečarić, T. Furuta, J. Mičić Hot and Y. Seo Mond-Pečarić Method in Operator Inequalities. Inequalities for Bounded Selfadjoint Operators on a Hilbert Space, Element, Zagreb, 2005.

16. M. Tominaga, Estimations of reverse inequalities for convex functions, Trends Math. 6, (2003), No.2, 129-139.

17. M. Tominaga, An estimate of quasi-arithmetic mean by arithmetic mean and its applications, Nikonhai Math. J. 17, (2006), 9-26. 
Faculty of Textile Technology, University of Zagreb, Prilaz baruna FilIPOVIĆA 28A, 10000 ZAGREB, CroAtia.

E-mail address: rozarija.jaksic@ttf.hr

E-mail address: pecaric@element.hr 